\title{
Hacia un análisis estructural del derecho a la salud y la igualdad: Con propósito de las medidas laborales adoptadas por el Estado ecuatoriano durante la pandemia del covid-19 en 2020
}

\author{
Towards a structural analysis of the right to health and equality: \\ In relation to the labor reforms adopted by the Ecuadorian State \\ during the Covid-19 pandemic in 2020
}

\author{
Fernando Bajaña Tovar (iD \\ Corte Constitucional del Ecuador
}

\begin{abstract}
RESUMEN Este artículo hace un recorrido sobre las principales corrientes liberales y sociológicas que sirven de soporte teórico para el análisis de la salud y de la igualdad en el derecho, con el fin de evidenciar la interrelación existente entre la concepción sociológica del derecho y la salud, y la perspectiva estructural del derecho y la igualdad. Para esto, se emplea un concepto de equidad sanitaria construida con los aportes de John Rawls sobre la justicia. Así, se evaluarán algunas de las medidas legislativas y ejecutivas adoptadas por el Estado ecuatoriano en materia laboral durante la pandemia del Covid-19 en 2020 y sus efectos en la capacidad de la población para costear servicios de salud.
\end{abstract}

PALABRAS CLAVE Igualdad estructural, equidad sanitaria, determinantes sociales, derecho a la salud, Covid-19.

ABSTRACT This article makes a tour of the main liberal and sociological currents that serve as theoretical support for the analysis of health and equality in the Law, in order to demonstrate the interrelation between the sociological conception of the right to health and structural perspective of the right to equality; using for this, the contributions of John Rawls on justice. Based on this, some of the legislative and administrative reforms adopted by the Ecuadorian State in labor matters will be evaluated during the Covid-19 pandemic in 2020 and its effects on the population's ability to pay for health services.

KEYWORDS Structural equality, health equity, social determinants, right to health, Covid-19. 


\section{Introducción}

En este ensayo se analizarán varias de las medidas laborales adoptadas por el Estado ecuatoriano durante la pandemia de Covid-19 en 2020, con base en los principios y subprincipios de la teoría de la justicia como equidad de John Rawls. En este sentido, se adaptarán los postulados de libertad e igualdad de la teoría de Rawls con el fin de proponer una tesis sobre la equidad sanitaria que sea útil para evaluar integralmente el fenómeno de la salud, particularmente en lo concerniente a la distribución de los bienes y servicios que son calificados como determinantes sociales de la salud (vivienda, agua, educación, etcétera).

Para esto, se empezará por exponer el contenido que las tesis liberales le han otorgado tradicionalmente al derecho a la salud y al derecho a la igualdad, identificándolos con categorías de salud ontológica, salud diagnóstica, igualdad formal e igualdad material, para luego dejar en evidencia que los postulados de dicha tesis resultan insuficientes para erigir un abordaje integral, preventivo y holístico de los problemas sanitarios en las sociedades.

Una vez sentadas estas conclusiones, se pasará revista por los distintos enfoques que han examinado a la igualdad como un fenómeno estructural con énfasis en la pobreza, la desigualdad y la equidad. Posteriormente, con base en los principios y subprincipios de equidad y diferenciación de la teoría de Rawls, se advertirá que no es posible un tutelaje completo del derecho a la salud si no se corrigen las posiciones desventajosas que afectan a las personas, provocadas por el azar genético y/o socioeconómico.

Una vez sentada esta base teórica, se evaluarán las políticas laborales adoptadas por el Estado ecuatoriano durante la pandemia del Covid-19 para la reducción de la jornada laboral de empleados privados y públicos, la forma en que dichas medidas repercutieron sobre los ingresos de las familias ecuatorianas y, en consecuencia, sobre la capacidad para costear bienes y servicios de salud.

Finalmente, se hará un recuento de los principales fallos de jurisprudencia vinculantes emitidos por la Corte Constitucional del Ecuador en materia de salud, con el fin de demostrar la manera en que la jurisprudencia de dicho órgano jurisdiccional ha evolucionado, acogiendo un enfoque cada vez más estructural y sociológico de la salud, y provocando que este organismo ocupe un rol importante tanto en la reglamentación como en la ejecución de políticas públicas equitativas en materia de salud.

\section{Tesis sobre el derecho a la salud}

Es posible identificar tres corrientes filosóficas en el abordaje del derecho a la salud, dos de índole liberal e individual que relacionan el concepto de salud tanto con el dominio psicosomático como con la ausencia de enfermedades, y una de corte sociológico, que evalúa la salud con base en los roles que cada persona cumple dentro de un grupo social, concentrándose en los efectos que traduce la posición psicosocial de las personas en su salud. De forma general, las aproximaciones liberales han sido clasificadas entre 
tesis ontológicas y diagnósticas, según partan de una definición positiva -como dominio- o negativa — como ausencia de patologías - de la salud.

\section{Tesis liberales ontológicas}

En el caso de las tesis ontológicas, destaca un claro predominio de los principios del liberalismo clásico con respecto a la libertad y la propiedad privada, concibiendo a la salud como «la capacidad del hombre para apropiarse y poseer su propio cuerpo» (Gracia, 1989: 32). Por consiguiente, estas tesis trazan un puente entre la salud de las personas y la protección de la libertad de cada individuo, y entre la propiedad del conjunto cuerpo-mente y el deber abstentivo del Estado de no afectar dicho derecho de propiedad, a partir de lo cual se derivarán cuatro importantes consecuencias con respecto al tratamiento jurídico de la salud.

Como primera consecuencia, al derecho a la salud se le asignará el carácter de un valor causal y no final, justiciándose su protección no por el hecho de configurar un fin en sí mismo, sino en la medida en que recrea un medio para la satisfacción de otros derechos y principios, especialmente el de libertad. Por lo tanto, un buen estado de salud física resulta deseable en la medida en que le permite a la persona ejercer la libertad de locomoción y de reposo, y los demás derechos que dependen de la activación de este binomio, como lo son el trabajo, la asociación, la protesta, la migración, etcétera. Asimismo, un buen estado de salud mental será preferible a uno deficiente, en cuanto brindará al individuo los medios necesarios para ejercitar su libertad de pensamiento y las facultades originadas en ella, a saber, la libertad de opinión, la objeción de conciencia, la autodeterminación de la personalidad, la expresión religiosa, entre otras.

Como segunda consecuencia, la calificación del estado de salud de una persona estará supeditada a la calidad de la apropiación que se verifique entre la persona y el conjunto de su cuerpo-mente. Así, una persona será saludable o tendrá un buen estado de salud si es que goza del completo dominio de su cuerpo objetivo o cuerpo desde fue$r a$, y controla suficientemente su cuerpo subjetivo o intracuerpo. En resumen, para la tesis ontológica, el contenido del derecho de propiedad, a saber, uso, goce y disposición de un bien, también sirve para explicar el contenido normativo del derecho a la salud y, más aún, la esencia de la persona en sí, toda vez que una persona saludable va a ser aquella que es y se tiene; y es (dimensión síquica) si domina su mente, y se tiene (dimensión somática) si hace lo propio con su cuerpo (Ortega y Gasset, 1983: 455).

Continuando con esta lógica, el hecho de que la salud sea reputada como una forma de propiedad sobre el conjunto cuerpo-mente traerá, como tercera consecuencia, que la tutela de la salud se circunscriba a una idea de libertad negativa, debido a la cual las instancias jurisdiccionales deberán velar por que no exista ninguna intromisión, embarazo o afectación que entorpezca la propiedad psicosomática de las personas, proveniente de cualquier factor externo, principalmente del Estado. Por ende, las disposiciones contenidas en los fallos de los jueces que conocen y resuelven sobre la violación del derecho a la salud se restringirán a confirmar la prohibición de turbar el complejo 
psicosomático de cada persona, y a asegurarse de que el dominio psicosomático de una persona retorne al estado previo a su perturbación.

Todo esto, a su vez, ocasiona que, como cuarta consecuencia, el deber del Estado, en lo que atañe a la salud, se configure de manera negativa, como una obligación de no hacer, o de abstenerse de turbar o lesionar el derecho de dominio que cada persona tiene sobre su cuerpo y mente, y de precautelar que todo tercero cumpla con esta abstención (deber de policía).

\section{Tesis liberales diagnósticas}

Las tesis diagnósticas, si bien mantienen un enfoque liberal para la explicación del concepto de salud y la justificación de su protección por parte del derecho, a diferencia de lo que sucede con la corriente ontológica, utiliza una noción de libertad configurada de manera positiva.

En efecto, la corriente diagnóstica, al equiparar la salud con ausencia de enfermedad, desencadena una relectura en clave prestacional de dicho derecho, haciendo que la obligación del Estado con respecto a la salud no se reduzca a un deber de abstención o de no afectación del conjunto psicosomático, sino que mude hacia una obligación de garantizar el acceso a la prestación y a la provisión de aquellos servicios y bienes sanitarios que permitan la curación y la rehabilitación de enfermedades.

Producto de este tránsito de paradigma de lo ontológico abstentivo a lo diagnóstico prestacional, la labor tutelar de los jueces y demás operadores jurídicos dejará de radicarse en la confirmación de la norma prohibitiva de no hacer o no afectar, y, en su lugar, estará direccionada a comprobar y corregir el acceso a servicios de atención sanitaria mediante el suministro de recursos y bienes de este tipo.

Sin perjuicio de esto, la tesis diagnóstica sigue teniendo un carácter individualista y situacional, en tanto que la obligación del Estado de proveer servicios sanitarios se activa una vez que haya existido una afectación comprobable en la salud de las personas (enfermedad), de ahí que no involucra necesariamente la provisión de servicios de prevención que tienen una mirada más sociológica y estructural.

A partir de lo expuesto, se puede observar que tanto las ideas individualistas que fundamentan las tesis ontológicas como las que sustentan las tesis diagnósticas carecen de un espectro tutelar amplio, y son inacabadas para explicar el fenómeno sanitario en su expresión social.

Por citar un ejemplo, si se considera que en el Ecuador el $71,1 \%$ de la población en áreas rurales se encuentra bajo el umbral de la pobreza multidimensional, esto es, que carece de acceso al agua por red pública, vive en pobreza extrema medida por ingresos $y$ en condiciones de hacinamiento, sin servicios de saneamiento de excretas y de recolección de basura, es poco probable que dicho sector de la población se encuentre interesado en que su derecho a la salud sea protegido por medio la inacción del Estado o su no intervención (libertad negativa). Contrariamente, lo más probable es que dichas personas deseen que las autoridades competentes los asistan mediante la prestación 
de un catálogo mínimo de servicios de salud (diagnóstico, tratamiento, medicación o rehabilitación), pero, principalmente, con la corrección de aquellas situaciones antes citadas (vivienda, pobreza, agua, etcétera), que no consideradas dentro del catálogo de servicios sanitarios, afectan gravemente al estatus sanitario de los individuos (Instituto Ecuatoriano de Estadísticas y Censos, 2019: 26).

Junto al individualismo, también es censurable que las tesis liberales funcionen bajo un mecanismo de acción caracterizado por una marcada tendencia a la restauración, en razón de la cual el contenido del derecho a la salud se limita a «restaurar» las situaciones vitales previas al acontecimiento de una enfermedad, atrofiando las aristas de prevención y el control epidemiológico de la salud.

La prevalencia de esta tendencia, en el plano jurisdiccional, ha causado que para la tutela del derecho a la salud se requiera que previamente haya existido algún tipo de lesión o enfermedad en una persona o grupo de personas singularizado o singularizable, lo que generalmente estará vinculado con algún tipo de falta de atención médica o provisión de medicamentos para el tratamiento de una patología o condición determinada.

Finalmente, las tesis liberales carecen de herramientas para abordar y dar respuesta a los problemas sanitarios que están vinculados a posiciones sociales o identidades de grupo, como la pertenencia a un sector étnico, el nivel de escolaridad de las personas, la situación de pobreza o la condición de empleo, en las que las medidas sanitarias a adoptar no se agotan en la abstención de intervenir del Estado o la provisión de servicios de salud, sino que demandan reformas estructurales en la sociedad. De esta forma, ni un paradigma de libertad positiva ni uno de libertad negativa encarnan herramientas epistemológicas y metodológicas útiles para explicar fenómenos empíricos que correlacionan el estado de salud de las personas con su entorno social.

\section{Tesis sociológica: Determinantes sociales de la salud}

Actualmente, existe evidencia consolidada que tiende a demostrar que la desigualdad afecta negativamente al estado de salud de los individuos, inclusive en niveles en los que la desigualdad no está acompañada de índices de pobreza o falta de acceso a bienes materiales. En este escenario, solo basta la desigualdad como dato empírico para verificar una suerte de gradiente social que patentiza una relación directa entre la posición social de las personas y su nivel de satisfacción sanitaria.

A partir de 1978, con la publicación de los estudios Whitehall, en cuyas conclusiones se expusieron los resultados del monitoreo que Michael Marmot, junto a su equipo, mantuvo por más de veinte años en torno a dieciocho mil empleados públicos del Reino Unido registrando las causas y sus fechas de muerte, además de los cargos que ocuparon dentro de la función pública, se pudo demostrar que existía «una marcada estratificación en la salud de la población estudiada, correlacionando el cargo ocupado y la tasa de mortalidad», y que «las personas en cargos jerárquicos tenían una tasa de mortalidad significativamente menor que las empleadas en cargos más bajos» (Segura del Pozo, 2014: 49-50). 
La metodología del estudio Whitehall pronto fue adaptada para investigaciones en ambientes no laborales, obteniéndose evidencia de que la correlación entre posicionamiento laboral y esperanza de vida también se percibía si se analizaban variables no económicas, como la raza, el género, la etnia o la ubicación geográfica. Por ejemplo, un estudio realizado por la Organización Panamericana de la Salud en Estados Unidos de América reveló que existían importantes brechas raciales, particularmente en el área de la salud materno-infantil. De esta manera, quedó en evidencia que «la tasa de mortalidad infantil, en los lactantes afroestadounidenses (13,3 por 1.000 nacidos vivos), equivalía a más del doble de las registradas en los lactantes blancos $(5,6)$ y en los lactantes hispanos $(5,5)$ », y que «la razón de mortalidad materna en las mujeres afroestadounidenses $(28,4$ por 100.000 nacidos vivos) representó, aproximadamente, el triple de las tasas registradas en las mujeres blancas $(10,5)$ y las mujeres hispanas $(8,9)$ » (Organización Panamericana de la Salud, 2012: 315).

Por otro lado, en el caso ecuatoriano, en ilustración de este fenómeno, las cifras del Instituto Nacional de Estadísticas y Censos de Ecuador (2018: cuadro 3.2.17) revelaron una importante relación entre el nivel de escolaridad y la falta de asistencia sanitaria al momento del parto, así como con la tasa de mortalidad general, de suerte que, en su mayoría, las mujeres $(41,5 \%)^{1}$ que no recibieron asistencia de personal médico durante las labores de parto eran de aquel grupo que no había terminado la instrucción secundaria, mientras que, en lo relativo a la tasa de mortalidad, en su mayoría, se concentró $(79,2 \%)$ en aquel sector de la población que no había accedido al bachillerato (Instituto Nacional de Estadísticas y Censos de Ecuador, 2018: gráfico 2.1.4). ${ }^{2}$

Este conjunto de variables económicas, sociales y culturales, con importante influencia en la salud de las personas (nivel de escolaridad, raza, etcétera), ha empezado a ser denominado como determinantes sociales de la salud, definiéndose a estos como todas aquellas condiciones «en las que las personas nacen, crecen, viven, trabajan y envejecen, y que afectan positiva o negativamente su morbilidad y esperanza de vida" (Organización Panamericana de la Salud y Organización Mundial de la Salud-Oficina Regional para las Américas, 2017).

En las dos últimas décadas, el interés institucional por el análisis de los determinantes sociales de la salud ha crecido progresivamente. De hecho, la Organización Mundial de la Salud, en 2005, inauguró la Comisión sobre Determinantes Sociales de la Salud con el objetivo de «acopiar datos para mejorar la salud y lograr que esté distribuida de forma más justa en todo el mundo, y para promover un movimiento mundial en favor de la consecución de esos objetivos». Por otra parte, la 62. ${ }^{a}$ Asamblea Mundial de la Salud (2009) aprobó la Resolución WHA62.14, en la que exhortaba a «reducir las inequidades sanitarias actuando sobre los determinantes sociales de la salud».

1. Cuadro denominado «Defunciones por lugar de residencia habitual, edad, sexo y nivel de instrucción».

2. Gráfico denominado «Nacidos vivos en el año ( $\mathrm{t}$ ) según nivel de instrucción de la madre y tipo de asistencia profesional». 
Sin embargo, la introducción de los determinantes sociales de la salud, como un nuevo elemento para analizar la salud de las personas, y la estrecha relación de estos con la categoría de desigualdad, origina una grave problemática para la epistemología de la salud que se sustenta en los enfoques liberales, ya que, tanto el criterio de salud, que identificaba a este concepto con el dominio psicosomático, y el que utiliza como parámetro de evaluación de la salud a la ausencia de enfermedades, están basados en concepciones de salud individual, que limitan la esfera de acción de este derecho, ya sea la no perturbación del individuo o al diagnóstico, tratamiento y rehabilitación de este. En consecuencia, todo esto es incompatible con una perspectiva en la cual el estado de salud de las personas está relacionado con su posición social y que, por tanto, las medidas de acción para la prevención, el mantenimiento y la restauración de la salud de los individuos necesariamente deben tener una proyección estructural dentro de un constructo social.

De ahí que se visualiza a las tesis sociológicas que advierten a la salud como una condición afectada socialmente, esto es, dependiente de los roles y de las tareas que ocupan las personas en una sociedad, y la forma en la cual se desarrollan los procesos de sociabilización, como aquellas que gozan de una mejor aptitud para abordar la problemática de los determinantes sociales de la salud y su estrecha relación con la desigualdad.

\section{Tesis sobre el derecho a la igualdad}

De manera semejante a lo detectado con el derecho a la salud, en lo que atañe al tratamiento y al desarrollo de la categoría de igualdad, el derecho se ha caracterizado por recibir influencias de corrientes liberales y sociológicas. Las corrientes liberales han sido las encargadas de brindar el campo teórico, a partir del cual se han originado los principales principios y dimensiones que se le atribuyen al derecho a la igualdad en la actualidad, desde la distinción entre igualdad material e igualdad formal, hasta la estandarización de los principios de no discriminación, y, más recientemente, de no sometimiento. Por su parte, las corrientes sociológicas son las responsables del naciente interés del derecho por los enfoques interseccionales de la igualdad, y la relectura valorativa de los fenómenos de desigualdad percatados en las sociedades con base en teorías de justicia como equidad.

\section{Igualdad formal y material}

En un inicio, las escuelas liberales, dentro del contexto de las revoluciones burguesas de finales del siglo XVIII e inicios del siglo XIX, identificaron el derecho a la igualdad con «la garantía de igualdad de trato a todos los destinatarios de la norma jurídica y la ausencia de todo privilegio en materia de jurisdicción e impuestos», correspondiéndole, por ende, al Estado un papel de protección negativa o de garante abstentivo, es decir, que debía cumplir con abstenerse de introducir distinciones injustificadas en las 
normas legales, y de garantizar que se mantengan vigentes las propiedades de impersonalidad y generalidad de la ley (Carmona Cuenca, 1994: 266).

Como resultado de esto, a los órganos jurisdiccionales se les asignaría el deber de corregir cualquier tipo de distinción normativa injustificada que cause efectos negativos a alguna persona o sector. De ahí que el origen del principio de no discriminación se encuentre ampliamente relacionado con la noción de igualdad ante la ley. En efecto, dicho principio procura sustraer del ordenamiento jurídico cualquier diferenciación realizada entre personas en igualdad de condiciones, que se compruebe como no motivada y que provoque consecuencias nocivas para la parte que es sujeto de la distinción.

No obstante, desde mediados del siglo XIX, con la aparición del Estado social de derecho y el predominio gradual de un enfoque más social en las tesis liberales, la idea de igualdad formal o igualdad ante la ley fue reinterpretada en clave material, incitándose a que se tuviera en cuenta la identidad de grupo de los sujetos. En palabras de Heller (1984: 322), «la igualdad formal de la democracia política, aplicada a situaciones jurídicas desiguales, producía un derecho material desigual», en razón de lo cual hacía falta que se dictaran medidas formalmente desiguales que permitieran corregir las situaciones económicas y sociales poco favorables que padecían ciertos sectores de la población.

De esta forma, con la percepción de una igualdad material, el Estado pasa a adoptar la posición de un garante positivo que debe encargarse de producir medidas que allanen los desfases sociales que se manifiestan en la población. Todo esto, a través de la tipificación de acciones afirmativas o medidas de discriminación inversa, esto es, a diferencia de lo que se analizaba en la igualdad formal, el Estado, para tutelar la igualdad material de sus ciudadanos, no solo deberá abstenerse de no patentar conductas discriminatorias, sino que deberá trabajar para que los efectos negativos provocados por el valor que se le da a ciertas categorías sociales que ubican de forma desventajosa a las personas dentro de la sociedad desaparezcan (sexo, raza, religión, condición migratoria, etcétera).

Por consiguiente, los jueces, dentro de su tarea jurisdiccional, pasan a estar obligados a admitir y reconocer como un mecanismo válido para la defensa de la igualdad y demás derechos humanos la vigencia de ciertas distinciones por medio de las cuales se le otorgue a los individuos de grupos históricamente afectados por la discriminación o exclusión una posición más ventajosa, surgiendo un principio general de no sometimiento, de manera análoga a lo advertido con el binomio de principio de no discriminación/derecho de igualdad formal.

No obstante, ambas perspectivas liberales del derecho a la igualdad no están indemnes de críticas y reparos, y de hecho suelen ser tachadas como individualistas y anecdóticas. De esta suerte, se le censura a la igualdad formal el que pueda servir para preservar normativamente el statu quo en la sociedad o, en otras palabras, que se preste para la perpetuación de la ventajas y desventajas de los diferentes grupos sociales, además de que predomine en ella un enfoque individualista que le otorgue a la igualdad un valor en exclusiva personal, lo cual trae consigo un papel omisivo del Estado, que debe 
garantizar que no exista ninguna intervención en ese valor individual de las personas.

Por su parte, suele criticársele a la tesis de igualdad material un formalismo implícito, un mecanismo de acción post injuriam y una prevalencia de lo anecdótico. Dicho de otro modo, se señala como algo negativo que la vigencia de las acciones afirmativas que defiende la igualdad material y el principio de no sometimiento se encuentre supeditada a las cargas formales de los procesos de configuración normativa (aprobación legislativa, emisión de reglamentos, etcétera), y que su tratamiento jurisdiccional solamente funcione cuando se haya demandado la existencia de un daño, sin incluir proyecciones preventivas, suscitándose, muchas veces, que la tarea de los jueces se limite a reponderar la categoría grupal que ha sido utilizada para la discriminación, dejando sin atender el resto de las categorías grupales que puedan identificar de forma interseccional a la víctima, y que pueden servir para discriminarla en otros ámbitos presentes o futuros.

Con esto, se dibuja una serie de puntos de encuentro entre las críticas que se hacen a las tesis liberales del derecho a la salud y a las del derecho a la igualdad, examinándose relaciones de paridad entre las tesis ontológicas de la salud y la tesis formal de la igualdad, y lo mismo entre la tesis diagnóstica de la salud y la tesis material de la igualdad. La tabla 1 permite contextualizar estas relaciones.

Tabla 1. Relaciones de paridad entre derechos a la salud y a la igualdad

\begin{tabular}{|l|l|}
\hline Salud ontológica & $\begin{array}{l}\text { Egualdad formal } \\
\text { Concepción del derecho como un valor individual. }\end{array}$ \\
\hline Igualdad material \\
\hline Salud diagnóstica & $\begin{array}{l}\text { No hay prevalencia de un enfoque preventivo. } \\
\text { La reparación del derecho es anecdótica y post injuriam. } \\
\text { Carece de una perspectiva holística e interseccional. }\end{array}$ \\
\hline
\end{tabular}

\section{Igualdad estructural}

Ante el escenario crítico que envuelve a las tesis liberales de la igualdad, las tesis sociológicas ofrecen una opción más estructural y sistémica del fenómeno de la igualdad, fundamentándolo directamente en las dinámicas de distribución de recursos y oportunidades. En este sentido, el contenido de derecho de igualdad no se verá configurado por la normalización del statu quo o la corrección de casos particulares de discriminación o sometimiento, sino en miras de la «dotación de recursos de todo tipo (educación, dinero, capital social, lengua, aspecto, etcétera), que dependen de la pertenencia a una u otra clase social, género o raza», y la necesidad de su moderación «por políticas estructurales que faciliten la inclusión en el mercado de trabajo» (Máiz, 2016: 19).

En relación con este aspecto, un punto de vista estructural de la salud no focalizará su objeto de tutela en el individuo, como sujeto de derecho (igualdad formal) o miem- 
bro de un grupo (igualdad material). En lugar de esto, se interesará principalmente en mecanismos de predistribución, que garanticen el igual acceso a las ventajas para todos y todas, incidiendo en «la manera en que el mercado distribuye los beneficios en primer lugar», y en mecanismos de redistribución vía sistema fiscal progresivo y pago de prestaciones (Máiz, 2016: 20).

De este modo, podría trazarse una línea de encuentro entre la visión estructural de la igualdad y la óptica de la salud basada en los determinantes sociales que ofrezca, en evidencia, una relación de doble implicación. Así, a través de la relación entre igualdad estructural y determinantes sociales de la salud, se hace posible abordar la forma en la cual estos determinantes (vivienda, agua, educación, etcétera) se encuentran distribuidos dentro de la sociedad, con el objetivo de corregir concentraciones y escaseces injustificadas (redistribuir), y la forma en la cual los servicios sanitarios, como un recurso autónomo, se encuentran distribuidos.

En esta línea, Gwatkin (2000: 6) propone evaluar la interacción del derecho de la salud con las dinámicas de distribución que estudia la igualdad estructural, con base en tres categorías: pobreza, igualdad y equidad. Todo esto, con el objetivo de «i) mejorar la salud de los pobres; ii) reducir las desigualdades en salud entre pobres y ricos; y iii) subsanar las desigualdades en salud».

En lo concerniente al objetivo de reducción de pobreza, el enfoque distributivosanitario propone la adopción de tácticas de crecimiento económico de abajo hacia arriba y de satisfacción de las necesidades humanas básicas como herramientas para garantizar la accesibilidad de los servicios y bienes sanitarios a las personas en un contexto de desarrollo sostenible. Por su parte, en lo que atañe al objetivo de reducción de las desigualdades en salud entre ricos y pobres, a la par de la línea de acción marcada por varias resoluciones y entidades adscritas a la Organización Mundial de la Salud, este mecanismo busca cerrar la brecha entre los que menos tienen y los que más tienen a efectos de asegurar la accesibilidad de los servicios de salud a todas las personas, sin distinción de su procedencia socioeconómica.

Sin embargo, tanto a los objetivos orientados a la reducción de la pobreza como a los dirigidos a la disminución de la brecha de desigualdad, se les refuta la carencia de supuestos epistemológicos y herramientas metodológicas ciertas, principalmente por la variedad de definiciones sobre pobreza y falta de homogeneidad en los usos estadísticos. Efectivamente, en el caso de la reducción de la pobreza existe una multiplicidad de definiciones y métodos cuantitativos que van desde la noción de "pobreza absoluta», empleada por el Banco Mundial, la cual usa un umbral uniforme de «ingresos/ consumo», y lo aplica a todas las personas sin distinción de su origen geográfico, hasta la concepción de "pobreza relativa», en la que el umbral de «ingresos/consumo» se encuentra adaptado a la realidad de un país determinado. Cosa semejante sucede con respecto a la desigualdad, particularmente en lo referente a su forma de medición, que, si bien generalmente se hace a través del coeficiente de Gini, que mide las diferencias económicas entre los distintos quintiles de una sociedad, se le critica a este su falta de multidimensionalidad. 
En este aspecto, la categoría de equidad se muestra como la más aceptada para comprender la relación entre igualdad estructural y determinantes sociales de la salud. La tesis de equidad parte como base de la idea de que en todas las sociedades existen grupos de personas que ocupan, una posición desfavorable, y que dicha posición no es una consecuencia racional de su conducta o atribuible a su responsabilidad, sino que se tiene como una desigualdad originaria y azarosa, como el haber nacido en un estrato pobre o pertenecer a una raza o etnia históricamente excluida. De ahí que la equidad, como virtud de las instituciones sociales, sea la encargada de corregir y mitigar estas desventajas originarias que afectan negativamente a las personas, valiéndose para esto del reordenamiento de la estructura social, política y económica con el objetivo de que todos los individuos sean considerados iguales, y gocen de equidad en el acceso a oportunidades de diversa índole, incluyendo a la asistencia sanitaria.

A propósito de esto, la opción por la equidad para el tratamiento estructural de la problemática de salud parece ser que ha sido adoptada por la Organización de Naciones Unidas desde inicios del nuevo milenio, como se puede ver de la Observación General 14 del Comité de Derechos Económicos, Sociales y Culturales de la Organización de Naciones Unidas sobre «el derecho al disfrute del más alto nivel posible de salud», la cual establece que:

Los establecimientos, bienes y servicios de salud deberán estar al alcance de todos. Los pagos por servicios de atención de la salud y servicios relacionados con los factores determinantes básicos de la salud deberán basarse en el principio de la equidad, a fin de asegurar que esos servicios, sean públicos o privados, estén al alcance de todos, incluidos los grupos socialmente desfavorecidos. La equidad exige que sobre los hogares más pobres no recaiga una carga desproporcionada en lo que se refiere a los gastos de salud en comparación con los hogares más ricos (Comité de Derechos Económicos, Sociales y Culturales de la Organización de Naciones Unidas, 2000: 4).

\section{Equidad sanitaria}

Entre las teorías de la equidad, probablemente sea la de John Rawls la más conocida y estudiada, y no es por menos, en cuanto logra conciliar los debates precedentes entre los postulados del liberalismo y el igualitarismo sin incurrir en una propuesta meramente utilitarista o intuitiva. Con esto, Rawls (2012: 73) plantea una teoría que emplea un principio de libertad y un principio de igualdad, a partir de los cuales una sociedad equitativa dependerá del cumplimiento de dos condiciones: que cada individuo miembro de esta sociedad tenga «el mismo derecho irrevocable a un esquema plenamente adecuado de libertades básicas iguales que sea compatible con un esquema similar de libertades para todos» (principio de libertad), y que las diferencias políticas, económicas y sociales agoten los requisitos de «estar vinculadas a cargos y posiciones abiertas a todos en condiciones de igualdad equitativa de oportunidades, y redunden un mayor beneficio de los miembros menos aventajados de la sociedad» (principio de igualdad). 
Cada una de estas dos condiciones configuran los principios que son pilares de la teoría de Rawls. Por medio de la primera, se busca garantizar una esfera de libertades individuales, cuya titularidad debe ser compartida por todos los individuos de la sociedad, revestida de un axioma de esencialidad que impide que ellas puedan ser negociables. De ahí que el primer principio de Rawls, si bien guarda similitudes con la concepción formal de la igualdad, como igualdad en la consideración normativa, se diferencia de esta en la medida en que no se limita a proteger la titularidad jurídica común de un catálogo de derechos y libertades, sino que, además, preserva a este catálogo dentro de las fronteras de lo innegociable.

Ahora bien, en lo que atañe al segundo principio de Rawls, es en este en el que realmente se empieza a visualizar, de forma directa, el plano estructural de su teoría, superando el plano formal del liberalismo clásico y enfocándose en la distribución equitativa de oportunidades y recursos. De este modo, para Rawls, la segunda condición para la construcción de una sociedad equitativa, a saber, el principio de igualdad, responde a una estructura dual, conteniendo en sí dos subprincipios: el de equidad de oportunidades, que busca superar los efectos del azar sobre el acceso igualitario de las personas a "las trayectorias profesionales y a todas las posiciones sociales relevantes», $y$ el de diferencia, que se funda en una estrategia maximin, y postula que siempre debe preferirse, en una sociedad, la maximización de las ventajas de los menos favorecidos (Luévano Cayón, 2018: 155).

En este orden de ideas, el principio de equidad de oportunidades propone superar la concepción limitada de igualdad formal que habían defendido los liberales clásicos, ya que, en ella, si bien existía una aparente garantía de acceso equitativo a las posiciones más altas de la estructura social (puntos de llegada), no se prestaba ninguna atención a las desigualdades sociales de origen (puntos de partida) provocadas por el azar genético (raza, etnia, genero, discapacidades, etcétera) o socioeconómico (pobreza, familia, ubicación geográfica, etcétera). De esta manera, el principio de equidad de oportunidades de Rawls busca contrarrestar las diferencias sociales que sirven para perpetuar las ventajas de ciertos sectores sobre otros. Esto provoca que, en una sociedad equitativa frente a la igualdad de talentos y disposición para ejecutarlos, debe corresponderle una igualdad de oportunidades en el acceso a los cargos y posiciones relacionadas a esos talentos, sin importar la procedencia social del titular.

En este sentido, es muy común que se le atribuya al subprincipio de equidad de oportunidades el ser el fundamento de muchas de las políticas públicas del Estado de bienestar, entre estas:

Las acciones públicas orientadas hacia el equilibrio en la distribución de la riqueza y el ingreso público a través de una política fiscal muy exigente, los programas de salud pública, la ampliación de las expectativas profesionales y vitales derivadas de un sistema educativo público e incluyente y la protección económica frente a las adversidades laborales como los sistemas de seguro de desempleo y pensiones son, en este contexto, los elementos característicos de este modelo de igualdad de oportunidades (Gutmann, 1980: 12). 
Por su lado, el subprincipio de diferencia emplea una interpretación productiva de la sociedad, identificándola con un sistema de cooperación productiva que permite que los individuos tengan una vida mejor a la que tuviesen si tuvieran que «tratar de vivir únicamente gracias a sus propios esfuerzos» (Rawls, 2012: 133). En esta línea, se empieza apreciar, en el principio de diferencia, una importante concentración en las dinámicas distributivas, en tanto que, al ser la sociedad para Rawls una estructura de cooperación productiva, todo lo que ahí se produzca deberá ser distribuido. Por lo tanto, este principio se enfocará sobre toda la estructura productiva y distributiva de la sociedad, y particularmente sobre las normas y autoridades que la organizan, incluyendo las reglas que rigen la división del trabajo, la asignación de roles y las escalas de salarios.

En resumidas cuentas, a través de este principio, Rawls materializa un aterrizaje realista de su teoría de la equidad, y da cuenta del hecho de que, como efecto del carácter limitado de los bienes que produce y dispone una sociedad, la distribución de estos tenderá a marcar diferencias entre los sectores que beneficia. Por ende, las reglas y autoridades que regulan la distribución deberán velar por que dichas diferencias estén ideadas de una forma que se beneficie principalmente a los que se encuentren en una situación de desventaja, es decir, siguiendo una estrategia de maximización de beneficios de las personas menos favorecidas o, en lenguaje matemático, adoptando una regla de maximin.

No obstante, Rawls no extiende el campo de acción del principio de diferencia para todos los bienes que tiene o produce una sociedad, sino que lo demarca en referencia a lo que denomina "bienes primarios", que, si bien es un concepto bastante indeterminado, lo define como esas cosas que necesitan y requieren las personas, vistas desde la óptica de la concepción política de la persona, esto es, como ciudadanos que son miembros plenamente cooperativos de la sociedad y no meramente como seres humanos al margen de cualquier concepción normativa (Rawls, 2012: 91).

Con motivo de lo analizado, una teoría de la equidad en la salud o equidad sanitaria deberá cumplir con defender:

- La protección normativa del derecho a gozar de prestaciones sanitarias a todas las personas de una sociedad, sin que dicho derecho sea transigible.

- La posibilidad de acceder de forma equitativa, a los servicios y bienes de salud a todas las personas, estableciéndose políticas públicas que corrijan los desfases negativos provocados por el azar o la situación de origen de cada sujeto.

- La adopción de medidas de diferenciación que beneficien principalmente a aquellas personas que se encuentran en una posición social o natural desventajosa.

Es así como, con fundamento en el subprincipio de equidad de oportunidades, en una teoría de equidad sanitaria, la salud sería el bien al cual los miembros de la sociedad tienen derecho a acceder, asignándosele, por tanto, el papel de un punto de llegada. No obstante, el punto de partida de cada individuo de la sociedad seguiría siendo la posición que ocupa en la escala social, y, en consecuencia, la corrección de dicha posición 
social no podrá efectuarse mediante políticas exclusivamente de provisión de servicios y bienes sanitarios, sino que deberá comprender todos aquellos factores que influyen en el posicionamiento social de un individuo, esto es, educación, trabajo, vivienda, pobreza o, en otras palabras, sobre los determinantes sociales de la salud.

Por su parte, en lo atinente al principio de diferencia, la estrategia maximin se concebiría tanto en relación con la distribución de bienes o servicios sanitarios, es decir, como un mecanismo de nivelación hacia arriba, cercano a lo analizado y propuesto por Gwatkin (2000: 12) para la disminución de defunciones de lactantes en Bolivia, Costa de Marfil e India, esto es, concentrando los recursos disponibles en el mejoramiento de los servicios sanitarios materno-infantiles de los quintiles más empobrecidos de la población, pero, a la vez, velando por no aumentar la tasa de defunciones de lactantes en los quintiles más altos; como en atención a todos los determinantes sociales de la salud, reproduciendo este mecanismo de nivelación hacia arriba en todos los aspectos que influyen en la salud (agua, vivienda, alimentación, etcétera), dejando nuevamente en evidencia la posibilidad de un vínculo entre la teoría estructural de equidad-propuesta y la tesis sociológica de la salud.

\section{Evaluación de medidas laborales adoptadas durante la pandemia del Covid-19: El caso ecuatoriano}

En el marco de la crisis provocada por la pandemia del Covid-19, el Ecuador ha adoptado diferentes medidas de naturaleza reglamentaria y legislativa. Entre estas medidas, destacan la aprobación de Ley Orgánica de Apoyo Humanitario para Combatir la Crisis Sanitaria Derivada del Covid-19 y la expedición de decretos y acuerdos ministeriales por parte del Ejecutivo que permiten establecer una jornada especial diferenciada de trabajo en el sector público, y que interpretan ciertas consecuencias provocadas por las pandemias con respecto a las relaciones laborales.

La Ley Orgánica de Apoyo Humanitario para Combatir la Crisis Sanitaria Derivada del Covid-19, que, según su preámbulo, fue concebida para «dar un alivio a la ciudadanía, al sector productivo y a la economía popular y solidaria para hacer frente a la actual situación económica y sanitaria del país», contiene disposiciones principalmente económicas, como la rebajas a pensiones educativas, la suspensión temporal de los trámites de desahucio en materia de inquilinato, el no incremento en los costos de servicios básicos y ciertas modificaciones a la estructura de las relaciones laborales. En este último aspecto, la Ley Orgánica de Apoyo Humanitario para Combatir la Crisis Sanitaria Derivada del Covid-19 introduce la figura de la reducción emergente de la jornada laboral, en razón de que se les da a los empleadores la facultad de «reducir la jornada laboral de los trabajadores hasta un máximo del 50\%».

Con respecto a este escenario, la reducción emergente de la jornada laboral introduce una considerable afectación a la estructura salarial de los trabajadores, en la medida en que, de conformidad con el artículo 20 de la ley mencionada: 
El sueldo o salario del trabajador corresponderá, en proporción, a las horas efectivamente trabajadas, y no será menor al 55\% del fijado previo a la reducción; y el aporte a la seguridad social debe pagarse con base en la jornada reducida.

En este sentido, la facultad de reducción de la jornada laboral que la Ley Orgánica de Apoyo Humanitario para Combatir la Crisis Sanitaria Derivada del Covid-19 le reconoce al empleador, y para la cual no es obligatoria ninguna etapa de consulta o consentimiento de los trabajadores, no solo tiene un impacto sobre las horas efectivamente trabajadas en una empresa, sino también sobre la cuantía de los sueldos de la masa laboral, y, en consecuencia, sobre la forma de distribución de los ingresos en las estructuras productivas (empresas).

En esta línea, si se sopesan las reformas laborales introducidas por la Ley Orgánica de Apoyo Humanitario para Combatir la Crisis Sanitaria Derivada del Covid-19, por medio del esquema de equidad sanitaria expuesto de forma previa, quedarán en evidencia varios indicadores adversos sobre la aplicación de los principios rawlianos de libertad e igualdad, especialmente de los subprincipios de equidad de oportunidades y diferencias.

De esta manera, si retomamos brevemente la definición del principio de libertad, se había dicho que este exigía como medio para la concreción de una sociedad justa «el mismo derecho irrevocable a un esquema plenamente adecuado de libertades básicas iguales que sea compatible con un esquema similar de libertades para todos» (Rawls, 2012: 73). De este modo, se puede afirmar que, en el marco constitucional ecuatoriano (Constitución de la República del Ecuador, 2008: artículo 328), ${ }^{3}$ se garantiza, dentro de ese «esquema plenamente adecuado de libertades básicas» para todos los trabajadores, el derecho a recibir un salario digno, básico, de aplicación general y obligatoria.

Con esto, en desarrollo del texto constitucional, el Código de Trabajo le encarga al Consejo Nacional de Salarios y al Ministerio de Trabajo la competencia para la fijación del monto de un salario básico, que, para 2020, mediante Acuerdo Ministerial DT2019-394, publicado el 27 de diciembre de 2019, se estableció en USD 400.

Por consiguiente, la distinción que introduce la Ley Orgánica de Apoyo Humanitario para Combatir la Crisis Sanitaria Derivada del Covid-19 atenta contra de la igualdad normativa de los trabajadores (principio de libertad), toda vez que faculta que cierto grupo de trabajadores sea sustraído del goce del salario mínimo digno que garantiza la Constitución por voluntad de sus empleadores.

En un lenguaje material, esto abre paso a que se creen profundas diferencias entre trabajadores de igual jerarquía y se profundicen las existentes entre los de distinto nivel jerárquico. Así, para el caso de los trabajos compensados con un salario mínimo vital (USD 400), los trabajadores que se vean afectados por la reducción de hasta un $45 \%$

3. La norma referida expresa: «La remuneración será justa, con un salario digno que cubra al menos las necesidades básicas de la persona trabajadora, así como las de su familia; será inembargable, salvo para el pago de pensiones por alimentos. El Estado fijará y revisará anualmente el salario básico establecido en la ley, de aplicación general y obligatoria». 
de sus ingresos salariales, producto de la reducción emergente de jornada, terminaría recibiendo un salario mensual de USD 220, alcanzado niveles de ingresos que no se reportaban desde 2009, cuando el salario mínimo vital fue de USD 218.

En la práctica, esto implicaría que haya trabajadores cumpliendo igualdad de funciones en ella o en diferentes organizaciones productivas, que no tengan igual derecho a trabajar una jornada completa, $y$, en consecuencia, a acceder a un salario que les permita cubrir sus necesidades básicas y las de su familia, incluyendo las relacionadas con la salud.

En esta línea, la evaluación de las posibilidades de sufragar gastos relacionados a la salud y sus determinantes, de los trabajadores afectados por la reducción emergente de la jornada laboral, si se la compara con las posibilidades de aquellos trabajadores que no son afectados por esta medida, demuestra una importante desventaja frente a los primeros. En este sentido, si se parte de las estimaciones del Instituto Nacional de Estadísticas y Censos de Ecuador (2012), los hogares ecuatorianos utilizan el 39,3\% de sus ingresos mensuales en gastos de salud y determinantes de la salud, como alimentación, agua y vivienda, destinando el 31,8\% para el sufrago de determinantes sociales (agua, vivienda, etc.) y el 7,5\% para servicios y bienes sanitarios propiamente dichos. Por ende, si se toma en consideración que los ingresos promedio de los hogares se calculan a partir de una estimación de 1,6 perceptores de ingresos, que ganan la remuneración básica unificada, según la metodología del Instituto Nacional de Estadísticas y Censos de Ecuador se concluirá que aquellos hogares en los que todos sus perceptores se vean afectados por la reducción emergente de la jornada laboral tendrán USD 113,18 menos para solventar gastos de salud, en relación con los hogares que no son afectados por esta medida (Instituto Nacional de Estadísticas y Censos de Ecuador, 2017).

Asimismo, se podrá visualizar que la capacidad total de gastos en salud de los hogares parcialmente afectados (USD 194,93) ni siquiera alcanza a la capacidad de gastos de los hogares sin afectación para determinantes sociales (USD 203,52). Finalmente, quedará en evidencia que la capacidad de gastos en bienes y servicios sanitarios de los hogares totalmente afectados será casi la mitad de la de los hogares sin afectación. Lo anterior se expresa en la figura 1 y en la tabla 2.

Es así como la reforma a las dinámicas laborales introducida por la Ley Orgánica de Apoyo Humanitario para Combatir la Crisis Sanitaria Derivada del Covid-19, en general, traduce un impacto negativo para la salud de los hogares, $y$, evaluada en clave de equidad sanitaria, se podría afirmar que implica una vulneración del principio de libertad, en la medida en que altera un derecho que se suponía garantizado de manera idéntica para todos los trabajadores: el salario básico vital. Todo esto, a su vez, deriva en una clara afectación a la capacidad de acceso a servicios y bienes sanitarios, así como la asunción de costos de servicios relacionados con determinantes sociales de la salud, como el agua, la vivienda y la alimentación.

En este orden de ideas, además de generarse una afectación al principio de libertad, se comprobaría la lesión del principio de equidad de oportunidades. Esto, en cuanto a 


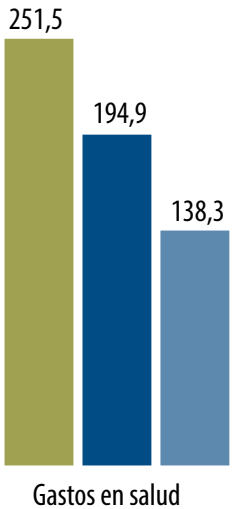

$(39,3 \%)$

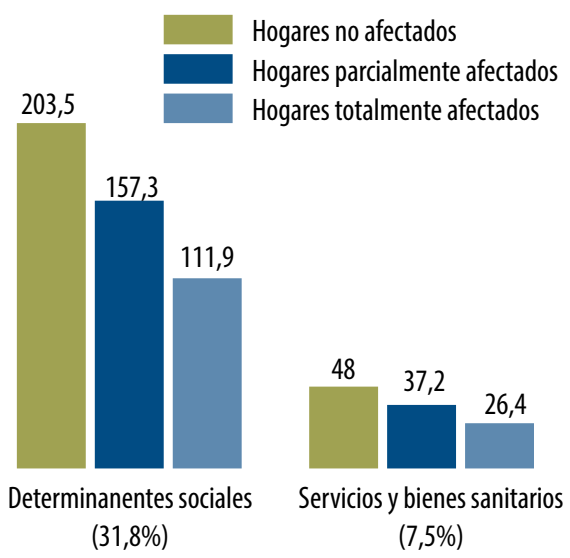

Figura 1. Impacto sobre la salud y sus determinantes sociales. Montos en dólares.

Tabla 2. Impacto sobre la salud y sus determinantes sociales

\begin{tabular}{|lccc|} 
& $\begin{array}{c}\text { Hogares no afectados } \\
\text { Gastos en salud (39,3\%) }\end{array}$ & $\begin{array}{c}\text { Hogares parcialmente } \\
\text { afectados }\end{array}$ & $\begin{array}{c}\text { Hogares totalmente } \\
\text { afectados }\end{array}$ \\
\hline Determinantes sociales (31,8\%) & 251,52 & 194,93 & 138,34 \\
\hline Servicios y bienes sanitarios (7,5\%) & 203,52 & 157,73 & 111,94 \\
\hline
\end{tabular}

que las posiciones sociales de aquellos individuos pertenecientes a los hogares afectados por la reducción emergente de la jornada laboral (puntos de partida) empeorarán, ubicándolos en desventaja para el goce de prestaciones sanitarias (punto de llegada).

No obstante, la alteración en los salarios mínimos vitales y la capacidad de gastos en salud no es la única consecuencia adversa que podría provocar la Ley Orgánica de Apoyo Humanitario para Combatir la Crisis Sanitaria Derivada del Covid-19, ya que, a largo plazo, las disposiciones que contempla, en lo referente a la seguridad social, principalmente el hecho de que bajo la modalidad de reducción eventual de jornada «el aporte a la seguridad social deberá pagarse con base en la jornada reducida», conforme a su artículo 20, terminará disminuyendo el nivel de la pensión que alcance con su jubilación y, por tanto, de manera general, en la capacidad de consumo, gastos y en las condiciones de vida durante la vejez.

Ahora bien, no solo las medidas legislativas son criticables desde la perspectiva de la equidad sanitaria, en cuanto se advierten también efectos negativos derivados de actos normativos de la función ejecutiva. En este sentido, el Acuerdo Ministerial MDT-2020$117,{ }^{4}$ que expide «las directrices para establecer la jornada especial diferenciada en el sector público», una figura semejante a la de la reducción emergente de la jornada labo-

\footnotetext{
4. Ministerio del Trabajo de Ecuador, Acuerdo Ministerial MDT-2020-117, disponible en https://bit. ly/3fyCizd.
} 
ral, contempla la posibilidad de aplicar esta medida a través de la cual se puede reducir la jornada de los trabajadores públicos, y, en proporción, el sueldo «hasta un límite no menor a treinta horas semanales», trayendo consecuencias en la salud similares a las estudiadas. Sin embargo, introduce ciertas excepciones, prohibiendo que se aplique esta medida a, entre otros, los profesionales del sector salud, lo cual parece razonable en consideración del contexto sanitario que se atraviesa, y «el personal activo de las Fuerzas Armadas y entidades que forman parte del Código Orgánico de las Entidades de Seguridad Ciudadana y Orden Público».

En este orden de ideas, el hecho de que se permita la suspensión de la jornada y la reducción salarial de los servidores públicos, exceptuando al personal de las Fuerzas Armadas y demás entidades de seguridad, no solo vulnera el principio de libertad, en la medida en que no se asegura el derecho de trabajo en igualdad de condiciones para los trabajadores del sector público, sino que, incluso, rompe el principio de diferencia, debido a que las distinciones que introduce (excepciones) están dirigidas a reforzar la estabilidad de un sector, que, más allá de no estar revestidos bajo la categoría de grupo prioritario en la Constitución, representan al ente de control y vigilancia del Estado, y, por tanto, ocupan una posición política ventajosa, como ostentador monopólico de la violencia legítima, lo cual va en contra del principio de Rawls, ya que las diferencias introducidas con respecto a la distribución de recursos y oportunidades no están sujetas a un criterio de maximin, beneficiando a un sector que ya ocupa una situación ventajosa, sin prestar atención a otros sectores que, además de ser considerados como prioritarios en la Constitución, ocupan situaciones desventajosas.

Finalmente, otro caso de rompimiento del principio de diferencia se puede advertir en los efectos provocados por el Acuerdo Ministerial MDT-2020-0815 (Ministerio del Trabajo de Ecuador, 2020a), que reformó al Acuerdo Ministerial MDT.2017-135 a través del cual se expidió el Instructivo para el Cumplimiento de las Obligaciones de los Empleadores Públicos y Privados, lo cual facilitó el registro de las desvinculaciones de trabajadores bajo la causal sexta del artículo 169 del Código de Trabajo (por caso fortuito o fuerza mayor que imposibiliten el trabajo), para la cual no se prevé indemnizaciones de despido sin la necesidad de una resolución judicial que confirme la concurrencia de esta situación. En efecto, a este acuerdo ministerial se le imputa el haber permitido la desvinculación laboral y desafiliación bruta de al menos 270.600 personas del seguro social, ${ }^{6}$ de las cuales, estimaciones para Latinoamérica del Banco Interamericano de Desarrollo, consideran que un $68,7 \%$ eran personas pobres. ${ }^{7}$ Todo esto sirve para

5. Ministerio del Trabajo de Ecuador, Acuerdo Ministerial MDT-2020-o81, disponible en https://bit. ly/3luscow.

6. «Desafiliaciones al IESS ya superan 270600 en cuatro meses de la pandemia del COVID-19», El Universo, 2 de agosto de 2020, disponible en https://bit.ly/37jeNiI.

7. «Resultados de encuesta sobre el coronavirus revelan importantes impactos, vínculos entre la desigualdad y los mercados laborales», Banco Interamericano de Desarrollo, 8 de mayo de 2020, disponible en https://bit.ly/3xw8sek. 
constatar nuevamente una mayor afectación a los estratos sociales más desfavorecidos, rompiéndose el criterio maximin.

\section{Control estructural del derecho a la salud: Una práctica naciente de la jurisprudencia constitucional ecuatoriana}

La jurisprudencia constitucional ecuatoriana, en lo atinente al derecho a la salud, ha venido experimentando un desarrollo progresivo transitando desde las tesis liberales hacia las sociológicas. Así, mientras que en las primeras sentencias de jurisprudencia vinculante sobre el derecho a la salud, emitidas en 2019, se detectaba una importante influencia de las tesis liberales, como en el caso Nole Ochoa con Instituto Ecuatoriano de Seguridad Social (sentencia de la Corte Constitucional del Ecuador, causa 904-12$\mathrm{JP} / 19,2019),{ }^{8}$ en la que las obligaciones de respeto y protección del derecho a la salud descifraban deberes de no hacer que recaían sobre el Estado y terceros, detectándose una clara influencia de las tesis ontológicas:

El derecho a la salud impone tres obligaciones generales: respetar, proteger y cumplir. La obligación de respetar exige que los Estados se abstengan de impedir directa o indirectamente en el disfrute del derecho a la salud. La obligación de proteger requiere que los Estados adopten medidas para impedir que terceros interfieran en la aplicación de las garantías previstas en el artículo. Por último, la obligación de cumplir requiere que los Estados adopten medidas apropiadas de carácter legislativo, administrativo, presupuestario, judicial o de otra índole para dar plena efectividad al derecho a la salud (sentencia de la Corte Constitucional del Ecuador, causa 904-12-JP/19, 2019: párr. 61).

O, como en el caso Santana Macías y otros (sentencia de la Corte Constitucional del Ecuador, causa 209-15-JH/19, 2019), ${ }^{9}$ en el que la Corte Constitucional del Ecuador afirmó que las autoridades competentes están obligadas a garantizar la disponibilidad y el alcance de la infraestructura, los bienes y los servicios sanitarios, en apego a las tesis diagnósticas:

Así, las autoridades competentes tienen la obligación de garantizar que los establecimientos, bienes y servicios de salud estén disponibles y al alcance de las personas privadas de libertad en los distintos centros de privación de libertad que integran el sistema de rehabilitación social a nivel nacional, garantizando un tratamiento médico adecuado que incluye, entre otras cosas, personal médico capacitado, medicamentos y equipo hospitalario científicamente aprobados y en buen estado, agua potable y condiciones sanitarias adecuadas (sentencia de la Corte Constitucional del Ecuador, causa 209-15-JH/19, 2019: párr. 37).

En 2020, la sentencia de jurisprudencia vinculante al caso Derecho a medicamentos de calidad, seguros y eficaces (sentencia de la Corte Constitucional del Ecuador, causa

8. Sentencia emanada el 13 de diciembre de 2019. Texto íntegro disponible en https://bit.ly/2TSnXiG. 9. Sentencia emanada el 12 de noviembre de 2019. Texto íntegro disponible en https://bit.ly/3xoKtxs. 
679-18-JP/20, 2020 $)^{10}$ introdujo un análisis sanitario de corte sociológico, inaugurando un análisis de la salud en relación con sus determinantes sociales al reconocer que:

Las personas están conectadas y determinadas por su entorno social, cultural y ambiental. Estos entornos condicionan y afectan la vida plena y saludable o la enfermedad. Las formas de producir alimentos, bienes, servicios y de consumir son factores importantes para la forma de vida saludable o para crear factores de riesgo para la salud (sentencia de la Corte Constitucional del Ecuador, causa 679-18-JP/20, 2020: párr. 46).

Este reconocimiento trajo consigo una percepción interseccional del papel de los órganos estatales con respecto a su deber tutelar frente al derecho a la salud, manifestando, en la misma sentencia, que la provisión de servicios hospitalarios, tratamientos médicos, prescripción y entrega de medicamentos para atender enfermedades es una concepción restringida y fragmentaria del derecho a la salud (sentencia de la Corte Constitucional del Ecuador, causa 679-18-JP/20, 2020: párr. 46).

Por este motivo, la política pública sanitaria «debe estar orientada y debe priorizar la prevención de la enfermedad y la promoción de entornos saludables para evitar la enfermedad» (sentencia de la Corte Constitucional del Ecuador, causa 679-18-JP/20, 2020: párr. 48). De ahí que este nuevo enfoque, además de sociológico, da paso a un entendimiento estructural del fenómeno sanitario, conforme con el cual el entorno en el cual se desarrolla la vida del individuo (situaciones socioeconómicas y ambientales) ocupa un lugar determinante en la protección de este derecho, surgiendo una clara vinculación entre salud e igualdad estructural:

Las políticas de salud no pueden formularse de forma aislada y dependen de otras políticas, como las ambientales, las de recursos naturales no renovables, las financieras, las educativas y más, que deben estar coordinadas y encaminadas desde el enfoque del derecho al disfrute del más alto nivel posible de salud (sentencia de la Corte Constitucional del Ecuador, causa 679-18-JP/20, 2020: párr. 49).

De este modo, la Corte Constitucional del Ecuador, además de mantener su clásico rol de máximo órgano de tutela de los derechos humanos, pasa a ocupar el rol de revisora y coautora de las políticas públicas del Estado sin agotar su análisis jurídico en lo anecdótico y a posteriori, velando porque se emplee un criterio interseccional, preventivo e interseccional de los derechos.

De hecho, en la sentencia en referencia, la Corte Constitucional del Ecuador fijó una serie de indicadores y metas que debían ser adoptados por el Estado ecuatoriano para la protección del derecho a la salud, entre los que destacan:

- El aseguramiento de un porcentaje mínimo del $80 \%$ de disponibilidad de medicamentos para cuidados paliativos que constan en la Guía de Cuidados Paliati-

10. Sentencia emanada el 5 de agosto de 2020. Texto íntegro disponible en https://bit.ly/3rRpqT8. 
vos (sentencia de la Corte Constitucional del Ecuador, causa 679-18-JP/20, 2020: párr. 282). ${ }^{11}$

- La creación de una partida presupuestaria desagregada para medicamentos del Cuadro Nacional de Medicamentos Básicos y para medicamentos fuera de él (sentencia de la Corte Constitucional del Ecuador, causa 679-18-JP/20, 2020: párr. 292). ${ }^{12}$

- La creación e implementación, por parte del Instituto Ecuatoriano de Seguridad Social, de un fondo solidario para enfermedades catastróficas (sentencia de la Corte Constitucional del Ecuador, causa 679-18-JP/20, 2020: párr. 292).

- La no regresividad en el número de visitas domiciliarias a pacientes que por su condición no pueden acceder a centro de salud (sentencia de la Corte Constitucional del Ecuador, causa 679-18-JP/20, 2020: párr. 282).

- La creación e implementación de un sistema de seguimiento nacional sobre medicamentos dispensados que están fuera del Cuadro Nacional de Medicamentos Básicos (sentencia de la Corte Constitucional del Ecuador, causa 679-18-JP/20, 2020: párr. 307). ${ }^{13}$

- La implementación de una historia clínica electrónica única para la RPIS, complementaria y de servicios (sentencia de la Corte Constitucional del Ecuador, causa 679-18-JP/20, 2020: párr. 307).

- La certificación de referencia internacional de la Agencia de Regulación, Control y Vigilancia Sanitaria del Ecuador, a fin de que alcance la calidad de autoridad de referencia regional de nivel 3 (sentencia de la Corte Constitucional del Ecuador, causa 679-18-JP/20, 2020: párr. 307).

Estos indicadores y metas, en el plano fáctico, configuran un ejercicio configurativo de la política pública sanitaria, competencia que, de forma general, ha estado en manos de la función ejecutiva del Estado (presidencia y ministerios), pasando a una autoridad con competencias jurisdiccionales como lo es la Corte Constitucional a participar en su control y reglamentación, lo que ha dado luces de una nueva forma de control judicial de este derecho, que supera el clásico mecanismo de acción post injuriam, en virtud del cual se avalaba que los órganos judiciales únicamente actuasen cuando se confirmase una lesión singular a la salud de un individuo, con el objetivo de adoptar un mecanismo de acción estructural, que, como tal, es general, preventivo y sistémico, lo cual facilita una comprensión multidimensional de la salud y sus determinantes sociales, y el análisis axiológico — deber ser - de la forma en que deben de distribuirse las oportunidades y recursos para la satisfacción de estos derechos.

\footnotetext{
11. Cuadro «Recepción del derecho».

12. Cuadro "Compromiso financiero».

13. Cuadro "Capacidad Institucional».
} 
Esta tendencia por un enfoque equitativo de la salud de la Corte Constitucional del Ecuador ha sido confirmada en la reciente sentencia de jurisprudencia vinculante 98318-JP/21 (sentencia de la Corte Constitucional del Ecuador, causa 983-18-JP/21, 2021), en la que dicho organismo confirmó los tres postulados para una teoría de equidad sanitaria, los cuales se abordarán a continuación:

\section{Protección normativa e innegociable del derecho a la salud}

En lo relativo a la accesibilidad, esta garantía exige la materialización de la igualdad en la esfera sanitaria. Debido a esto, no basta con que todas las personas tengan acceso a la atención sanitaria en un sentido formal, es decir, en un sentido netamente normativo (titularidad). Es imperativo que también se garantice el acceso en condiciones de igualdad en un orden real y material (accesibilidad) (sentencia de la Corte Constitucional del Ecuador, causa 983-18-JP/21, 2021: párr. 124).

\section{Acceso igualitario a bienes y servicios sanitarios}

En lo tocante a la disponibilidad, esta garantía busca que el Estado asegure la suficiencia de infraestructura, recursos humanos, bienes y servicios para la prestación de los servicios de salud y el mantenimiento de la salubridad en la sociedad, lo que demanda que el Estado deba adoptar mecanismos de inversión y provisión progresiva de fondos para desarrollar sus sistemas de asistencia sanitaria (sentencia de la Corte Constitucional del Ecuador, causa 983-18-JP/21, 2021: párr. 117).

\section{Estrategias de diferenciación con criterio maximin}

Por su parte, la accesibilidad económica, de conformidad con la Observación General 14 del Comité de Derechos Económicos Sociales y Culturales de la Organización de Naciones Unidas, asegura que los establecimientos, bienes y servicios de salud deberán estar al alcance de todos. Los pagos por servicios de atención de la salud y servicios relacionados con los factores determinantes básicos de la salud deberán basarse en el principio de la equidad, a fin de asegurar que estos servicios, sean públicos o privados, estén al alcance de todos, incluidos los grupos socialmente desfavorecidos. La equidad exige que sobre los hogares más pobres no recaiga una carga desproporcionada, en lo que se refiere a los gastos de salud, en comparación con los hogares más ricos (sentencia de la Corte Constitucional del Ecuador, causa 983-18-JP/21, 2021: párr. 133).

\section{Conclusiones}

Como se ha revisado durante el desarrollo de este artículo, entre las tesis liberales que se han formulado para la comprensión del contenido del derecho a la salud destacan la tesis ontológica y la tesis diagnóstica, que entienden al derecho a la salud, ya sea como dominio o propiedad de la estructura psicosomática, ya sea como ausencia de 
enfermedad, lo cual, en relación con la figura del Estado, verificará en este un rol de garante omisivo o de garante prestacional en dinámicas curativas y de rehabilitación. Como parangón, las tesis liberales sobre el derecho a la igualdad han observado en esta tanto un mecanismo para la protección de la igualdad formal o igualdad ante la ley, con su correlativa prohibición de discriminación normativa, como un mecanismo de discriminación positiva que busca el no sometimiento de ciertos grupos humanos. No obstante, a las tesis liberales del derecho a la salud y del derecho a la igualdad se le critican, en el ámbito jurídico, estar caracterizadas por actuar a través de dinámicas post injuriam y situacionales, esto es, que favorecen a un contenido normativo que opera únicamente cuando se ha verificado la violación del derecho de un individuo particular, careciendo de un enfoque estructural, preventivo y sistémico.

Por su parte, la tesis sociológica de la salud esboza una interpretación interseccional del fenómeno sanitario, en la que ocupan un lugar central los determinantes sociales de la salud, todas aquellas situaciones en la que las personas se desarrollan y que afectan a su esperanza de vida, como la pobreza, la vivienda, el nivel de educación, entre otras. Como su contrapartida, en el estudio del derecho a la igualdad, entre las tesis que abogan por una igualdad estructural, la idea de inspiración rawliana de equidad sanitaria se observa como la mejor alternativa para la tutela material de la salud como fenómeno complejo y multidimensional. En efecto, la concreción de una tesis de equidad sanitaria exigiría:

- La protección normativa del derecho a la salud con carácter intransigible -principio de libertad.

- El acceso igualitario a los servicios y bienes de salud a todas las personas (principio de igualdad).

- Adoptar medidas de diferenciación que beneficien principalmente a aquellas personas que se encuentran en una posición social o natural desventajosa (principio maximin).

En el caso ecuatoriano, se observó que algunas de las medidas legislativas y administrativas adoptadas para paliar los efectos de la pandemia por el Covid-19, particularmente la Ley Orgánica de Apoyo Humanitario para Combatir la Crisis Sanitaria Derivada del Covid-19 y los acuerdos ministeriales MDT-2020-117 y MDT-2020-081, si bien rigen sobre materia laboral, regulando la forma de suspensión y diferenciación de jornadas de trabajo, así como la forma de desvinculación de trabajadores, terminan repercutiendo, de manera determinante, sobre el derecho a la salud de las personas en consideración a la relación existente entre los ingresos por salarios básicos de las personas y la capacidad para acceder a servicios y bienes sanitarios, además del costeo de otros determinantes sociales de la salud, como agua potable, vivienda, educación, etcétera.

Para finalizar, se expuso la práctica naciente en la jurisprudencia vinculante de la Corte Constitucional del Ecuador, en la que se ha adoptado un rol activo en la formu- 
lación de las políticas públicas sanitarias, con la disposición de medidas estructurales, preventivas y sistémicas que debe cumplir la función ejecutiva en la programación estatal de la protección del derecho a la salud, lo cual evidenciaría la posibilidad de una transición del control constitucional clásico, como medio de tutela de derechos humanos, interpretación y aplicación de las constituciones, hacia un control constitucional estructural, en el que las políticas de las Cortes Constitucionales superarían la reducción a un esquema jurisdiccional tradicional y acogerían un nuevo esquema que, si bien jurisdiccional, tomaría partida en las dinámicas administrativas con miras en la protección preventiva y sistémica de los derechos humanos.

\section{Referencias}

Asamblea Mundial de la Salud (2009). Resolución WHA62.14: Reducir las inequidades sanitarias actuando sobre los determinantes sociales de la salud. Disponible en https://bit.ly/3ywYhYg.

Carmona Cuenca, Encarnación (1994). «El principio de igualdad material en la jurisprudencia del Tribunal Constitucional». Revista de Estudios Políticos (Nueva Época), 84: 265-286. Disponible en https://bit.ly/3lydrIW.

Comité De Derechos Económicos, Sociales y Culturales de la Organización de Naciones Unidas (2000). Observación General 14. Disponible en https://bit.ly/3AfckBQ.

Gracia, Diego (1989). «De la higiene privada a la salud pública: Beneficios y riesgos de la política de salud». Razón y Fe: Revista Hispanoamericana de Cultura, 1089-109o: 27-40.

Gutmann, Amy (1980). Liberal Equality. Nueva York: Cambridge University Press.

Gwatkin, Davidson R. (2000). «Desigualdades sanitarias y salud de los pobres: ¿Qué sabemos al respecto? ¿Qué podemos hacer?». Boletín de la Organización Mundial de la Salud. Recopilación de artículos, 3: 3-17. Disponible en https://bit.ly/3rgAtJo.

HeLler, Hermann (1984). Las ideas socialistas. Madrid: Alianza.

Instituto ECuatoriano de Estadísticas y Censos (2012). Encuesta Nacional de Ingresos y Gastos de los hogares urbanos y rurales (2011-2012). Quito: Instituto Nacional de Estadística y Censos (INEC). Disponible en https://bit.ly/316Mt39.

-. (2017). Ficha metodológica de la canasta básica familiar. Quito: Instituto Nacional de Estadística y Censos (INEC). Disponible en https://bit.ly/2Zwzffu.

-. (2018). Anuario de estadísticas vitales. Quito: Instituto Nacional de Estadística y Censos (INEC) - Presidencia de la República del Ecuador.

-. (2019). Encuesta Nacional de Empleo, desempleo y subempleo (ENEMDU), marzo 2019. Quito: Instituto Nacional de Estadística y Censos (INEC). Disponible en https://bit.ly/3jlRkCR.

LuÉvano CaYón, Ana Regina. (2018). Justicia sanitaria: Las teorías de la justicia distributiva. Tesis Doctoral, Universidad Complutense de Madrid-Facultad de Ciencias Políticas y Sociología. Disponible en https://bit.ly/3xpYxyO. 
MÁiz, Ramón (2016). «De la economía a la ética, ¿Qué fue de la Política? Para una teoría estructural de la igualdad». Revista de Estudios Políticos, 174: 13-46. Disponible en https://bit.ly/3CiyMfl.

Organización Panamericana de la Salud y Organización Mundial de la Salud-Oficina Regional para las Américas (2017). Determinantes sociales de la salud en la región de las Américas. Disponible en https://bit.ly/3A3qpT4.

Organización Panamericana de la Salud (2012). Situación salud en las Américas 2012: Panorama regional y perfiles de país. Washington D. C.: Organización Panamericana de la Salud.

Ortega y Gasset, José (1983). «Vitalidad, alma, espíritu». En Obras completas. Vol. 2. Madrid: Alianza.

Rawls, John (2012). La justicia como equidad. Barcelona: Paidós/Estado y Sociedad. SEGURA DEL Pozo, Javier (2014). «Las desigualdades sociales en salud: un reto para las políticas públicas». Zerbitzuan: Gizarte zerbitzuetarako aldizkaria, 55: 47-63. Disponible en https://bit.ly/3leBpb3.

\section{Sobre el autor}

Fernando Bajaña Tovar es abogado por la Universidad Católica de Santiago de Guayaquil. Actualmente ejerce como asesor del despacho de la jueza constitucional Teresa Nuques en la Corte Constitucional del Ecuador. Su correo electrónico es fbajana95@ gmail.com. (D) https://orcid.org/0000-0002-1622-1479. 
